\title{
Eccentric connectivity index of chemical trees
}

\begin{abstract}
The eccentric connectivity index $\xi \mathrm{c}$ is a distance-based molecular structure descriptor that was recently used for mathematical modelling of biological activities of diverse nature. We prove that the broom has maximum $\xi \mathrm{c}$ among trees with a fixed maximum vertex degree, and characterize such trees with minimum $\xi$ c. In addition, we propose a simple linear algorithm for calculating $\xi \mathrm{c}$ of trees
\end{abstract}

Keyword: 